\title{
Impact of Histopathological Diagnosis with Ancillary Immunohistochemical Studies on Lung Cancer Subtypes Incidence and Survival: A Population-Based Study
}

\author{
Andrea Bordoni, ${ }^{1}$ Massimo Bongiovanni, ${ }^{2}$ Luca Mazzucchelli, ${ }^{2}$ and Alessandra Spitale ${ }^{1}$ \\ ${ }^{1}$ Ticino Cancer Registry, Institute of Pathology, 6600 Locarno, Switzerland \\ ${ }^{2}$ Division of Clinical Pathology, Institute of Pathology, 6600 Locarno, Switzerland
}

Correspondence should be addressed to Andrea Bordoni, andrea.bordoni@ti.ch

Received 26 September 2011; Accepted 7 November 2011

Academic Editor: Carmen J. Marsit

Copyright (C) 2011 Andrea Bordoni et al. This is an open access article distributed under the Creative Commons Attribution License, which permits unrestricted use, distribution, and reproduction in any medium, provided the original work is properly cited.

\begin{abstract}
Purpose. The aim of this study was to assess the impact of immunohistochemical- (IHC-) studies on incidence and survival of lung cancer histotypes. Patients and Methods. Lung cancers occurred in southern Switzerland between 1996 and 2010 were selected by the Ticino Cancer Registry and categorised into adenocarcinoma (AC), squamous-cell-carcinoma (SqCC), small-cell-carcinoma (SmCC), and large-cell carcinoma/non-small-cell lung cancer (LCC/NSCLC). Incidence rates, annual-percentage-change (APC), and two-year overall survival (OS) (follow-up: 31.12.2010) were performed. Results. 2467 cases were selected: 997 (40.4\%) AC; 522 (21.2\%) LCC/NSCLC, 378 (15.3\%) SmCC, and 570 (23.1\%) SqCC. Trend-analysis showed significant increase in AC (APC: 4.6; 95\% CI: 3.1; 6.0) and decrease of LCC/NSCLC, with significant joinpoint in 2003 (APC: - 14.7; 95\% CI: -21.6; -7.1). Improved OS and decreased OS were detected in SqCC and LCC/NSCLC, respectively. Conclusions. This study highlights that diagnosis with ancillary immunohistochemical studies will change incidence and survival of precisely defined lung cancer subtypes. It calls attention to the need for cautious interpretation of studies and clinical trials, where the diagnosis was based on histology unaccompanied by IHC studies, and to the need of standardised diagnostic procedures.
\end{abstract}

\section{Introduction}

Lung cancer is one of most common cancers in the world, representing $17.1 \%$ of all cancers in men, $6.7 \%$ in women, and $12.2 \%$ in both sexes [1]. Of the histological types, adenocarcinoma (AC) has remained the most prevalent among women over the past three decades, with incidence rates increasing slowly over time in many countries. In contrast, squamous cell carcinoma (SqCC) has historically been the predominant tumour type in men, but the incidence has declined and converged with the corresponding incidence in women, which has remained fairly stable [2].

Traditionally, lung carcinoma was classified into histological types using standard histological techniques. The most critical step in histopathological diagnosis was to distinguish small cell carcinoma (SmCC) from the other lung carcinomas, which were collectively called the non-small cell lung carcinomas (NSCLCs); patients with the former were referred to chemotherapy, whereas patients with the latter were potentially eligible for surgery or different chemotherapies. Over the past few years, the emergence of targeted or combination treatment strategies has created new demands on histopathological diagnostics, as it is now recognised that the efficacy and toxicity of some new drugs are related to the histological type [3]. Consequently, the exact determination of histological type by a pathologist has become essential to making clinical decisions [4].

In this context, the integration of conventional histomorphological analysis with an immunohistochemical (IHC) panel that includes markers of squamous (i.e., p63, cytokeratin (CK) 5/6) and glandular (TTF-1, CK7) cell differentiation allows more accurate identification of the histotype [5-12]. Moreover, recent studies have demonstrated that the antibody panel approach may help to refine lung cancer 
classification in fine needle aspiration (FNA) cell blocks as well as in biopsy material; remarkably, this approach reduces the proportion of cancers diagnosed as NSCLCs and thus improves the quality of treatment decisions $[11,13]$.

The aims of this study were as follows: (i) to assess changes in lung cancer incidence in the south of Switzerland after the progressive introduction from 2003 to 2009 of ancillary IHC studies for the diagnosis of lung cancers (panel systematically applied from 2010) and (ii) to evaluate potential changes in short-term survival trends of the four major lung cancer histotypes.

\section{Material and Methods}

2.1. Case Selection. All patients with invasive lung cancer diagnosed by cytology or histology in the south of Switzerland between 1996 and 2010 were selected from the files of the population-based Ticino Cancer Registry. The Registry is located in the southern, Italian-speaking region of Switzerland and is closely connected to and part of the regional Institute of Pathology, which serves the entire region [14].

Lung tumour topography and lung tumour histotypes were classified using the International Classification of Diseases for Oncology (ICD-O-III) and the WHO Classification of Tumours of the Lung $[15,16]$. The tumour site was classified according to the extended ICD-O version proposed by Wagner [17]. Case registration and comparability, validity, and consistency checks were performed according to the International Agency for Research on Cancer (IARC) guidelines and the recommendations of the European Network of Cancer Registries (ENCRs) [18-20].

Lung cancers were categorised into four histotypes: AC, SqCC, SmCC, and large cell carcinoma/non-small-cell lung cancer (LCC/NSCLC) $[15,16]$. We included NSCLC, not formally classified by WHO, in the LCC histotype, as considered by default "poorly differentiated" tumours, resulting in the group called LCC/NSCLC [16]. Carcinomas not otherwise specified (NOS), lymphomas, neuroendocrine tumours (i.e., carcinoids and large cell neuroendocrine carcinomas), and mesenchymal tumours were excluded from the present study. Tumours were staged according to the Cancer Staging Manual of the American Joint Committee on Cancer (AJCC), version 5, until 2002 and according to version 6 from 2003 onwards. The tumours were then categorised as "limited disease" (any T, any $\mathrm{N}$, and M0) or "extensive disease" (any T, any N, and M1) [16, 21, 22].

All histological evaluations were made at the local Institute of pathology by the same core group of pathologists. IHC studies for histotyping lung cancers (i.e., TTF-1, p63, CK 5/6 and 7) were progressively introduced from 2003 to 2009. Since 2010 a diagnostic algorithm is applied to all new cases systematically.

2.2. Statistical Analysis. Mean and median values are provided for quantitative variables; proportions represent qualitative variables. Differences among lung cancer histotypes were evaluated using 1-way analysis of variance (ANOVA) for patient age (years). A chi-square test was conducted to assess the relationship between the different lung cancer histotypes and main clinicopathological characteristics such as sex, histological grade, and tumour extent at diagnosis. The Kruskal Wallis test was used to compare ordinal variables such as age groups.

European age-standardised incidence rates were calculated by the direct standardization method to remove the strong correlation between patient age and tumour occurrence [23]. Trends in incidence were measured as the estimated annual percentage change (APC) and the 95\% confidence interval (CI), which were calculated by fitting a linear regression to the natural logarithm of the age-adjusted rates $(r)$, with year of diagnosis as the regressor variable as follows: $\ln (r)=m$ (year) $+b$. From $m$, the slope of the regression line, the APC was calculated as follows: APC = $100 \times\left(e^{m}-1\right)$ [24-26]. Incidence trends were analysed by lung cancer histotypes for both genders together. Statistical significance was set at $P<0.05$. Two-year overall survival (OS) (follow-up on December 31, 2010) was analysed for patients with a 24-month complete follow-up (incidence period 1996-2008) using the Kaplan-Meier method. The logrank test was used to detect statistically significant differences in survival. The two-year survival trend analysis considered three incidence periods: 1996-2001 (before the introduction of the IHC panel), 2002-2004 (transitional period), and 2005-2008 (after the introduction of the complete IHC panel). The statistical analysis was conducted in SAS version 9.1 (SAS Institute Inc, Cary, NC, USA).

\section{Results}

Of 2844 lung cancers that occurred in the south of Switzerland between 1996 and 2010, 2585 (90.9\%) were confirmed by cytological or histological assay and were therefore selected for inclusion in the present study. The 118 cases that did not meet the inclusion criteria (i.e., carcinomas NOS, lymphomas, neuroendocrine, and mesenchymal tumours) were excluded from the analysis, and the remaining 2467 cases constitute the basis of the present study. The prevalence of each of the four lung cancer histotypes was as follows: 997 (40.4\%) ACs, 522 (21.2\%) LCC/NSCLCs, 378 (15.3\%) SmCCs, and 570 (23.1\%) SqCCs.

Table 1 summarises the main clinical-pathological characteristics of the patients. The four lung cancer histotypes differed significantly by mean age at diagnosis (ANOVA $F$-test $=7.49, P<0.0001), \mathrm{AC}$ being dominant in the youngest group $(66.0 \pm 11.0$ years old). When subjects were stratified by age group, this result was confirmed $(P<$ $0.0001)$. Overall, most lung cancers occurred in men $(69 \%)$, with a pick of $80.5 \%$ in SqCC $(P<0.0001)$. In terms of histological grade at diagnosis, $62.6 \%$ of AC tumours and $54.2 \%$ of SqCC tumours were poorly differentiated $(P=$ 0.0048). A significant difference was found when lung cancer histotypes were compared by tumour extent at diagnosis $(P<0.0001)$ : SqCC showed the highest prevalence of limited disease $(77.2 \%)$, whereas $>50 \%$ of SmCCs were classified as "extensive". We also detected an overall significant difference $(P<0.0001)$ in the distribution of histotypes across the two 


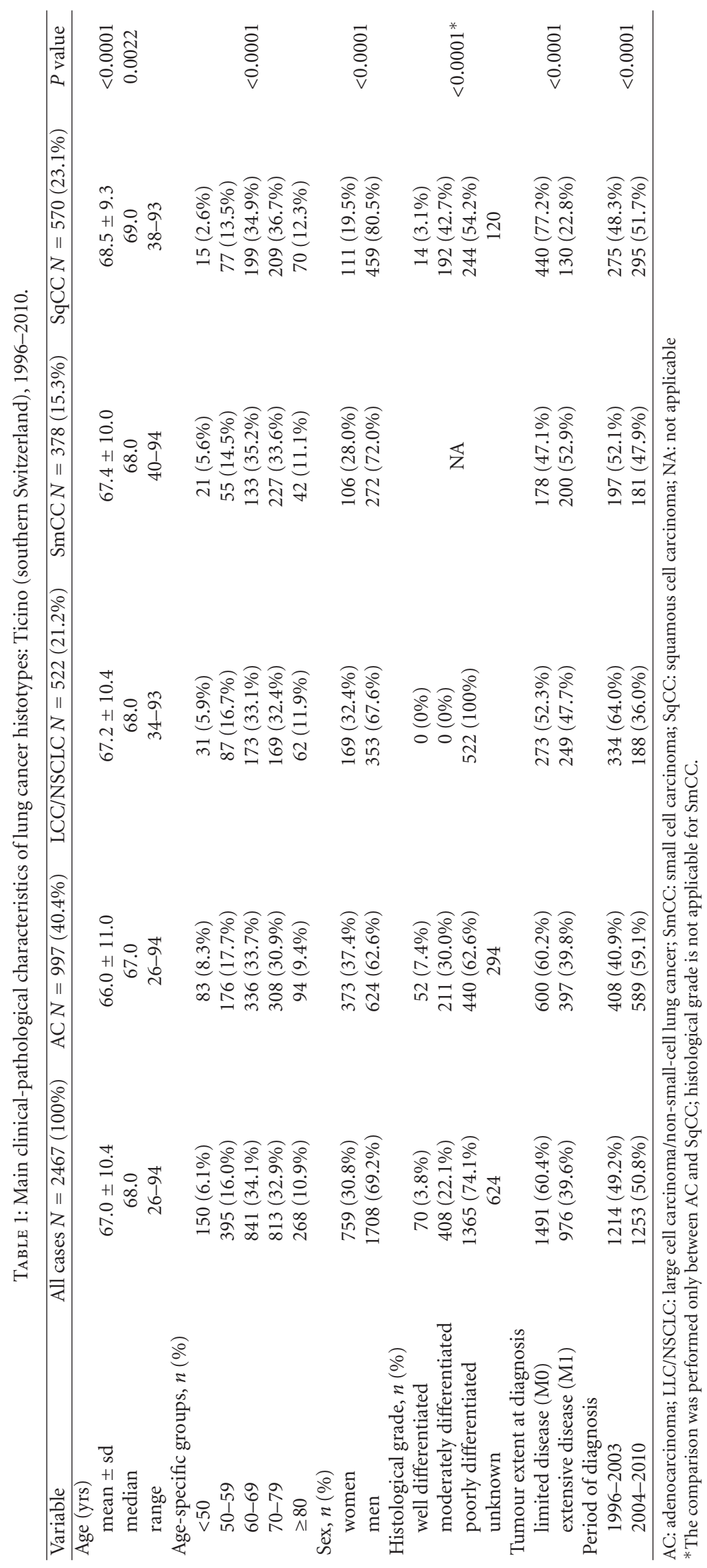




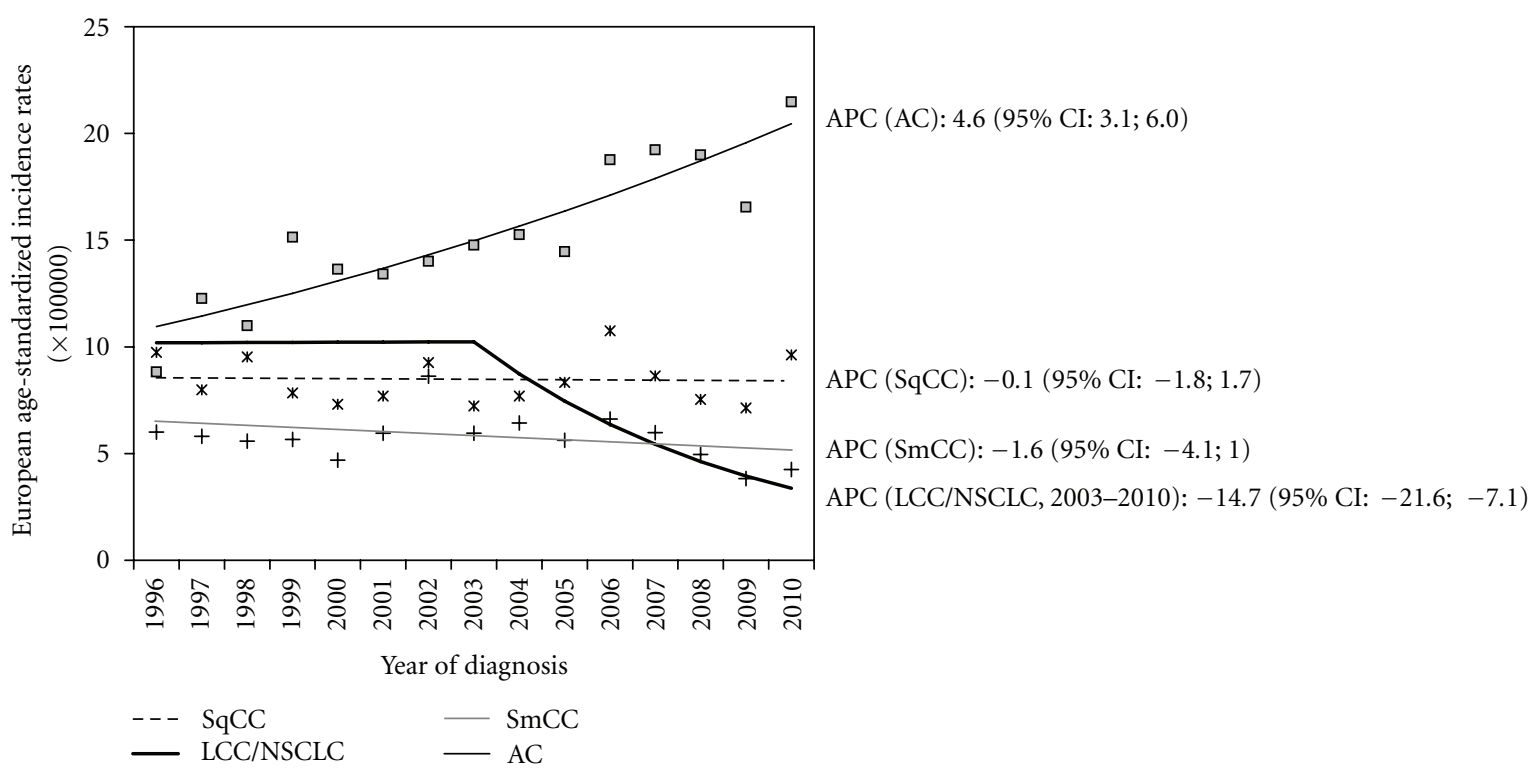

AC: adenocarcinoma; LLC/NSCLC: large-cell carcinoma/non-small-cell lung cancer SmCC: small-cell carcinoma; SqCC: squamous cell carcinoma

FIGURE 1: Trend of European age-standardised incidence rates of lung cancer histotypes: Ticino, 1996-2010.

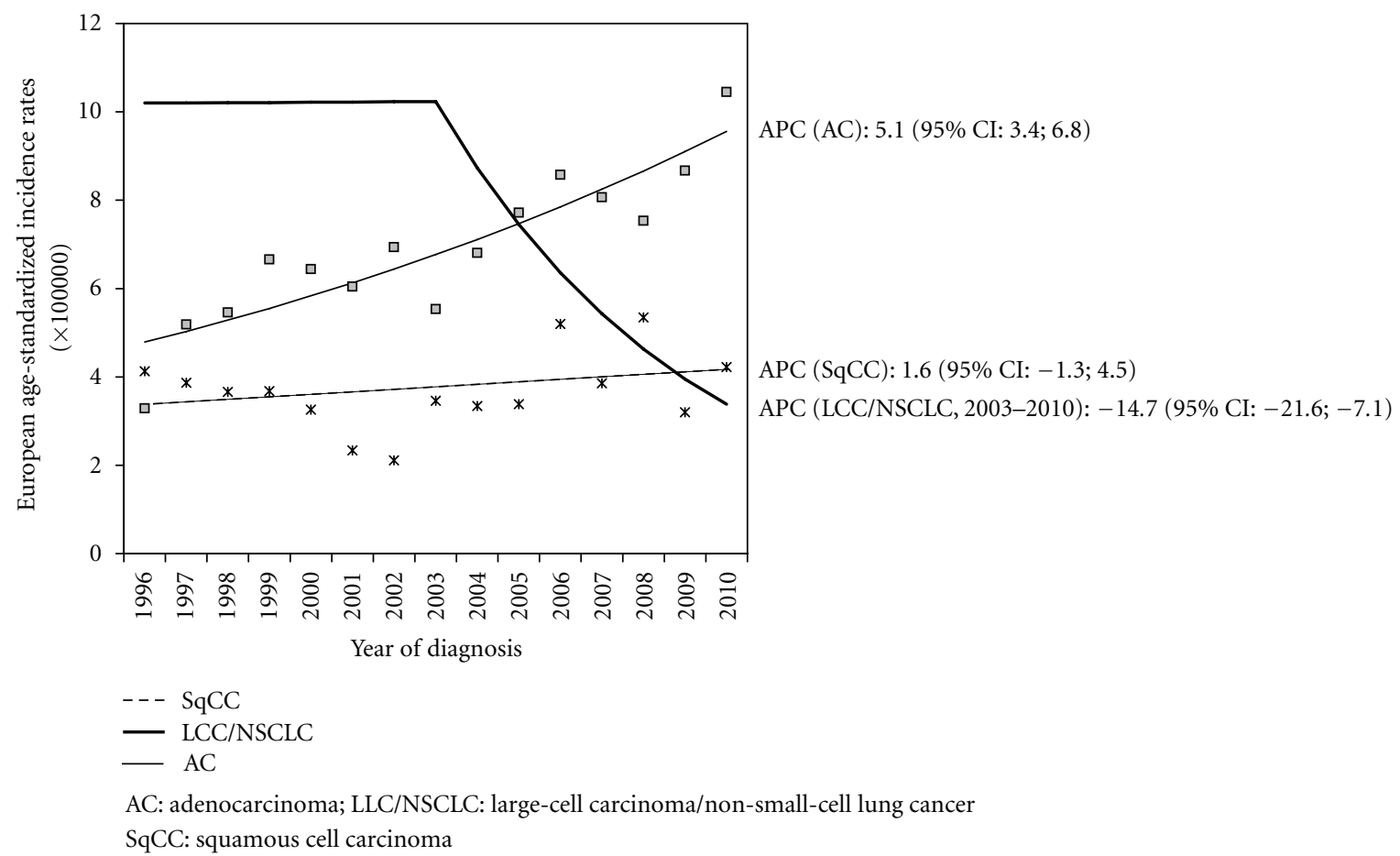

FIgURE 2: Trend in European age-standardised incidence rates of poorly differentiated lung cancer histotypes: Ticino, $1996-2010$.

study periods (Table 1). In particular, we observed a higher proportion of AC tumours after 2003 (40.9\% in 1996-2003 versus $59.1 \%$ in $2004-2010, P<0.0001$ ), corresponding to a percentage increase of $44.5 \%$ of the total cases; in contrast, there was a significant decrease in LCC/NSCLC proportion (64.0\% in $1996-2003$ versus $36.0 \%$ in $2004-2010, P<$
$0.0001)$, corresponding to a percentage decrease of $43.8 \%$. The prevalence of SmCC and SqCC remained stable.

The above-described trends of proportions were confirmed by the trend analysis of incidence rates, which show a significant increase in AC incidence (Figure 1, APC: 4.6; 95\% CI: $3.1 ; 6.0$ ) accompanied by a decrease in LCC/NSCLC 
incidence, with a significant joinpoint in 2003 (APC: - 14.7; $95 \%$ CI: $-21.6 ;-7.1)$. We also detected a slight decrease in the incidence of SqCC and SmCC, but it was not statistically significant. The trend seen in AC was also observed when we considered only poorly differentiated lung cancers (APC: 5.1; 95\% CI: 3.4; 6.8) (Figure 2).

Short-term OSs by lung cancer histotype are reported in Figure 3; SmCCs and LCC/NSCLCs show the worst survival probabilities at two years from diagnosis $(P<0.0001)$. We also detected an increase in OS for SqCC and a decrease for LCC/NSCLC, particularly in the last study period, 2005-2008 (Figure 4).

\section{Discussion}

The observed baseline increase in the incidence of $\mathrm{AC}$ and decrease in the incidence of SqCC, which were observed in both genders, are similar to the trends observed worldwide and described in many studies [27-29]. The decreased incidence of smoking in men, the increased incidence of smoking in women, accompanied by changes in the composition of cigarettes, and the implementation of filters are suggested to be responsible for the observed changes $[2,30]$. It has also been postulated that the use of filtered cigarettes has caused an increase in the incidence of $\mathrm{AC}$, which is usually located in the peripheral lung, because smokers now need to inhale more deeply to achieve a comparable effect. The exposure of the lung tissue to tobacco smoke is thus more extensive [31].

In addition to the increasing incidence of $\mathrm{AC}$, our study highlights a significant decrease in the incidence of the LCC/NSCLC histotype, with a joinpoint in 2003. This observation is difficult to explain by changes in exposure to known lung cancer risk factors. More likely it is associated with the progressive introduction (beginning in 2003) of a panel of IHC markers for squamous (i.e., p63, CK 5/6) and glandular differentiation (TTF-1, CK7). Many studies have already described the benefit to diagnosis of a specific panel of markers that can help pathologists to correctly classify lung cancer histotypes [32-34]. In particular, major benefits are obtained in distinguishing poorly differentiated SqCC from AC, especially in small biopsy tissues or cell specimens obtained by bronchus brossage/lavage or FNA $[6-9,35]$. In recent years, a precise histopathological classification of lung carcinomas has become increasingly important as a way to identify candidates for targeted therapy and to improve the design of clinical trials $[3,12]$. Thus, the observed decrease in LCC/NSCLC diagnosis and increase in AC diagnosis will help to better define the target populations that could benefit from these new drugs.

Concerning potential confounding factors, it is unlikely that WHO's 2003 changes in lung cancer classification (1996-2002, according to the second edition of the International Classification of Diseases for Oncology (ICD-OII); 2003-2010, according to the third edition (ICD-O-III)) significantly influenced our results. Before the introduction of ICD-O-III in 2003, LCC and NSCLC were routinely considered together as a poorly defined and poorly differentiated entity (since 2003 the NSCLC code was not available in ICDO codes, and as consequence LCC diagnosis was treated as a

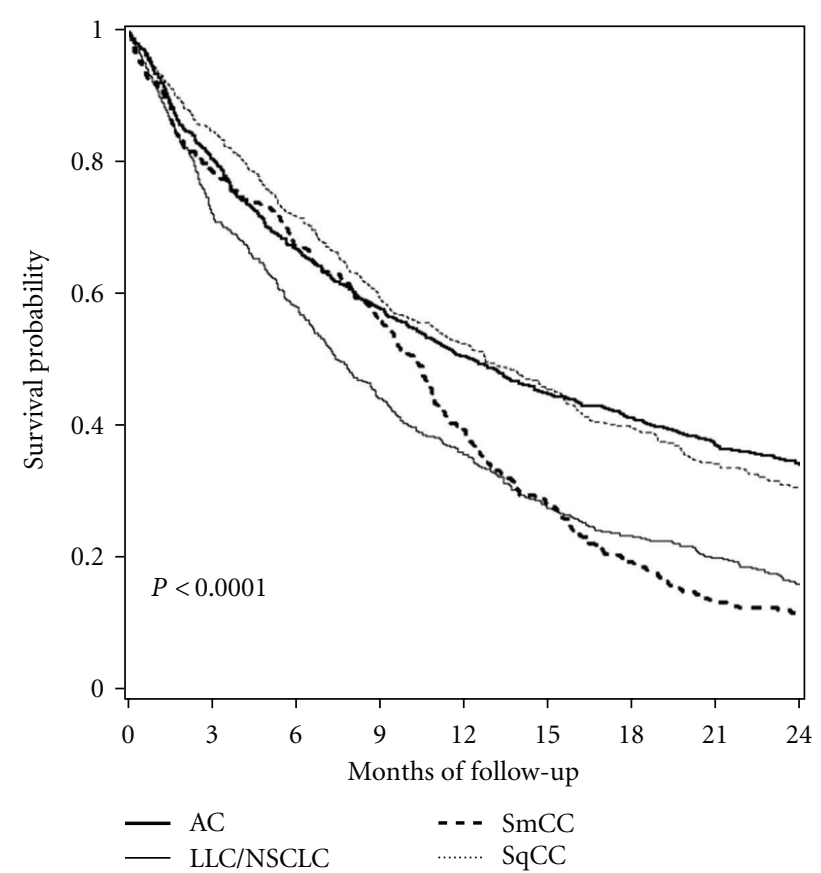

AC: adenocarcinoma; LLC/NSCLC: large-cell carcinoma/nonsmall-cell lung cancer

SmCC: small-cell carcinoma; SqCC: squamous cell carcinoma

Figure 3: Short-term overall survival by lung cancer histotypes: Ticino, 1996-2008.

"catch-all"). In order to avoid classification bias, we pooled these two groups in the second study period as well. Another potential confounding factor (not observed in our study, data not shown) would be a shift along the study period in diagnostic procedure (i.e., cytology versus histology), which could cause an increase or decrease in diagnostic accuracy.

The significant changes in incidence by histological subtype appear to be followed by changes in the short-term OS. This trend, which is most evident for LCC/NSCLC and SqCC, is important, as it could have affected the results of some clinical trials, particularly in cases where the diagnosis of lung cancers was based on histological evaluation without ancillary studies, or where ancillary studies were progressively introduced over the study period. In this contest, the worst short-term OS for LCC/NSCLC in the last period of the study (Figure 4) most likely reflects the selection of poorly differentiated carcinomas. Additional studies are warranted to clarify this observation as well as the improved short-term OS of patients with SqCC that may be related to a general improvement of cancer care.

The most significant aspects of this study are the following: (i) it includes an up-to-date case study (1996-2010) composed of a large number of lung cancer cases, thus reflecting a representative real-world description of a population with universal and homogeneous access to treatment; (ii) all ages were considered, thereby ensuring the observation of a total population at risk; (iii) the panel analyses of diagnostic markers were all carried out by a single laboratory and evaluated on routinely collected tissue by the same core 


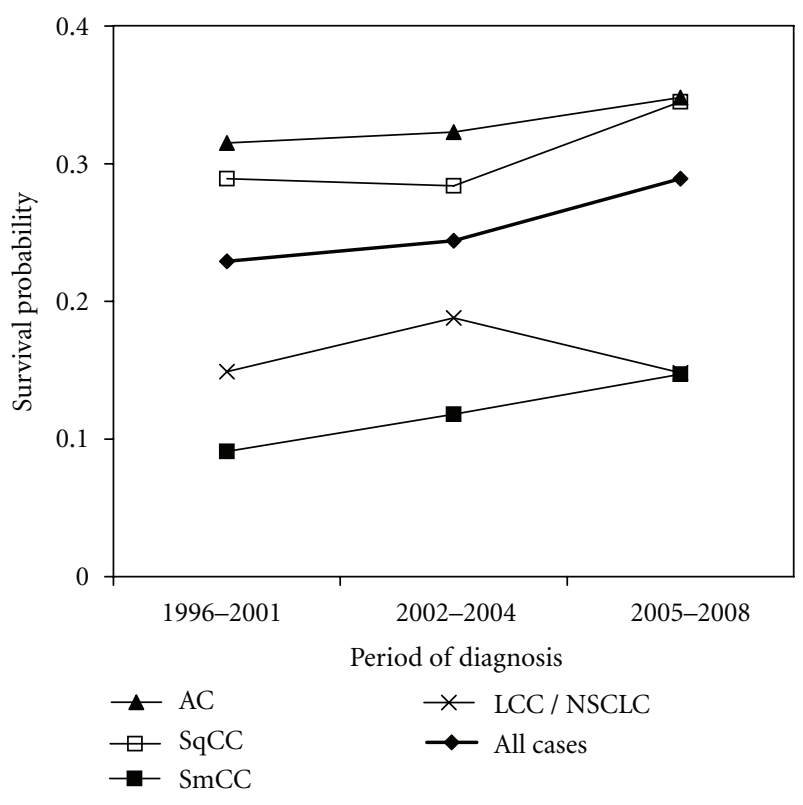

AC: adenocarcinoma; LLC/NSCLC: large-cell carcinoma/nonsmall-cell lung cancer

SmCC: small-cell carcinoma; SqCC: squamous cell carcinoma

Figure 4: Trend in two-year overall survival by lung cancer histotypes: Ticino, incident cases in the periods 1996-2001, 20022004, and 2005-2008.

group of pathologists, thus ensuring the reproducibility of results.

In conclusion, in this population-based study we observed a statistically significant decrease of LCC/NSCLC histotype, which is most likely attributable to the introduction of IHC diagnostic markers. We underscore the need for cautions interpretation of previous studies and clinical trials in which the diagnosis of lung cancer was only based on conventional histomorphological evaluation. In the era of targeted therapy, we advocate not only quality control programs for molecular testing but also a standardisation of histological diagnoses.

\section{Conflict of Interests}

The authors indicate that there are no potential conflict of interests.

\section{References}

[1] J. Ferlay, H.-R. Shin, F. Bray, and D. Forman, GLOBOCAN 2008: Cancer Incidence and Mortality Worldwide, IARC CancerBase No. 10, International Agency for Research on Cancer, Lyon, France, 2008.

[2] B. L. Egleston, S. I. Meireles, D. B. Flieder, and M. L. Clapper, "Population-Based Trends in Lung Cancer Incidence in Women," Seminars in Oncology, vol. 36, no. 6, pp. 506-515, 2009.

[3] C. J. Langer, B. Besse, A. Gualberto, E. Brambilla, and J.C. Soria, "The evolving role of histology in the management of advanced non-small-cell lung cancer," Journal of Clinical Oncology, vol. 28, no. 36, pp. 5311-5320, 2011.
[4] W. D. Travis, E. Brambilla, M. Noguchi et al., "International association for the study of lung cancer/American Thoracic Society/European Respiratory Society international multidisciplinary classification of lung adenocarcinoma," Journal of Thoracic Oncology, vol. 6, no. 2, pp. 244-285, 2011.

[5] G. Rossi, G. Pelosi, P. Graziano, M. Barbareschi, and M. Papotti, "Review article: a reevaluation of the clinical significance of histological subtyping of non-small-cell lung carcinoma: diagnostic algorithms in the era of personalized treatments," International Journal of Surgical Pathology, vol. 17, no. 3, pp. 206-218, 2009.

[6] M. Wu, B. Wang, J. Gil et al., "p63 and TTF-1 immunostaining: a useful marker panel for distinguishing small cell carcinoma of lung from poorly differentiated squamous cell carcinoma of lung," American Journal of Clinical Pathology, vol. 119, no. 5, pp. 696-702, 2003.

[7] B. Y. Wang, J. Gil, D. Kaufman, L. Gan, D. S. Kohtz, and D. E. Burstein, "p63 in pulmonary epithelium, pulmonary squamous neoplasms, and other pulmonary tumors," Human Pathology, vol. 33, no. 9, pp. 921-926, 2002.

[8] H. A. Harlamert, "Thyroid transcription factor-1 and cytokeratins 7 and 20 in pulmonary and breast carcinoma," Acta Cytologica, vol. 42, no. 6, pp. 1382-1388, 1998.

[9] N. G. Ordóñez, "Thyroid transcription factor-1 is a marker of lung and thyroid carcinomas," Advances in Anatomic Pathology, vol. 7, no. 2, pp. 123-127, 2000.

[10] P. A. Bejarano, R. P. Baughman, P. W. Biddinger et al., "Surfactant proteins and thyroid transcription factor-1 in pulmonary and breast carcinomas," Modern Pathology, vol. 9, no. 4, pp. 445-452, 1996.

[11] E. C. McLean, H. Monaghan, D. M. Salter, and W. A. Wallace, "Evaluation of adjunct immunohistochemistry on reporting patterns of non-small cell lung carcinoma diagnosed histologically in a regional pathology centre," Journal of Clinical Pathology, vol. 64, no. 12, pp. 1136-1138, 2011.

[12] A. G. Nicholson, D. Gonzalez, P. Shah et al., "Refining the diagnosis and EGFR status of non-small cell lung carcinoma in biopsy and cytologic material, using a panel of Mucin staining, TTF-1, cytokeratin 5/6, and P63, and EGFR mutation analysis," Journal of Thoracic Oncology, vol. 5, no. 4, pp. 436441, 2010.

[13] S. Mukhopadhyay and A. L. A. Katzenstein, "Subclassification of non-small cell lung carcinomas lacking morphologic differentiation on biopsy specimens: utility of an immunohistochemical panel containing TTF-1, napsin A, p63, and CK5/6," American Journal of Surgical Pathology, vol. 35, no. 1, pp. 15$25,2011$.

[14] A. Bordoni, A. Spitale, and P. Mazzola, "Ticino Cancer Registry," 2009, http://www.ti.ch/cancer.

[15] A. Fritz, A. Jack, D. M. Parkin et al., International Classification of Diseases for Oncology, World Health Organization, Geneva, Switzerland, 3rd edition, 2000.

[16] W. D. Travis, E. Brambilla, and C. C. Harris, Pathology and Genetics of Tumours of the Lung, Pleura,Thymus and Heart, World Health Organization, Lyon, France, 2004.

[17] G. Wagner, Tumor-Lokalisationsschlüssel: International Classification of Diseases for Oncology ICD-O, 2. Topographischer Teil, Springer, Berlin, Germany, 5th edition, 1993.

[18] O. M. Jensen, D. M. Parkin, R. MacLennan, C. S. Muir, and R. G. Skeet, Cancer Registration: Principles and Methods, IARC Scientific Publication No. 95, IARC, Lyon, France, 1991.

[19] J. E. Tyczynski, E. Démaret, and D. M. Parkin, Standards and Guidelines for Cancer Registration in Europe. The ENCR 
Recommendations, vol. 1 of IARC Technical Publication n.40, IARC, Lyon, France, 2003.

[20] D. M. Parkin, V. W. Chen, J. Ferlay, J. Galceran, H. H. Storm, and S. L. Whelan, "Comparability and quality control in cancer registration," IARC Technical Report No 19, IARC, Lyon, France, 2004.

[21] I. D. Fleming, J. S. Cooper, D. E. Henson et al., AJCC Cancer Staging Manual, Lippincott-Raven, Philadelphia, Pa, USA, 5th edition, 1997.

[22] F. L. Greene, D. L. Page, I. D. Fleming et al., AJCC Cancer Staging Manual, Springer, New York, NY, USA, 6th edition, 2002.

[23] J. Esteve, E. Benhamou, and L. Raymond, Statistical Methods in Cancer Research, vol. 4 of IARC Scientific Publications, World Health Organisation, Lyon, France, 2004.

[24] D. Kleinbaum, L. Kupper, and K. Muller, Applied Regression Analysis and Other Multivariable Methods, Duxbury Press, North Scituate, Mass, USA, 1988.

[25] "Statistical Research and Applications Branch and National Cancer Institute," Joinpoint Regression Program, Version 3.0., 2005.

[26] H. J. Kim, M. P. Fay, E. J. Feuer, and D. N. Midthune, "Permutation tests for joinpoint regression with applications to cancer rates," Statistics in Medicine, vol. 19, no. 3, pp. 335351,2000 .

[27] A. Charloux, E. Quoix, N. Wolkove, D. Small, G. Pauli, and H. Kreisman, "The increasing incidence of lung adenocarcinoma: Reality or artefact? A review of the epidemiology of lung adenocarcinoma," International Journal of Epidemiology, vol. 26, no. 1, pp. 14-23, 1997.

[28] M. L. G. Janssen-Heijnen and J. W. W. Coebergh, "The changing epidemiology of lung cancer in Europe," Lung Cancer, vol. 41, no. 3, pp. 245-258, 2003.

[29] S. S. Devesa, F. Bray, A. P. Vizcaino, and D. M. Parkin, "International lung cancer trends by histologic type: male:female differences diminishing and adenocarcinoma rates rising," International Journal of Cancer, vol. 117, no. 2, pp. 294-299, 2005.

[30] M. J. Thun, C. A. Lally, J. T. Flannery, E. E. Calle, W. D. Flanders, and C. W. Heath, "Cigarette smoking and changes in the histopathology of lung cancer," Journal of the National Cancer Institute, vol. 89, no. 21, pp. 1580-1586, 1997.

[31] S. D. Stellman, J. E. Muscat, S. Thompson, D. Hoffmann, and E. L. Wynder, "Risk of squamous cell carcinoma and adenocarcinoma of the lung in relation to lifetime filter cigarette smoking," Cancer, vol. 80, no. 3, pp. 382-388, 1997.

[32] L. Righi, P. Graziano, A. Fornari et al., "Immunohistochemical subtyping of nonsmall cell lung cancer not otherwise specified in fine-needle aspiration cytology: a retrospective study of 103 cases with surgical correlation," Cancer, vol. 117, no. 15, pp. 3416-3423, 2011.

[33] N. Rekhtman, D. C. Ang, C. S. Sima, W. D. Travis, and A. L. Moreira, "Immunohistochemical algorithm for differentiation of lung adenocarcinoma and squamous cell carcinoma based on large series of whole-tissue sections with validation in small specimens," Modern Pathology, vol. 24, no. 10, pp. 1348-1359, 2011.

[34] G. Pelosi, G. Rossi, F. Bianchi et al., "Immunhistochemistry by means of widely agreed-upon markers (cytokeratins 5/6 and 7 , p63, thyroid transcription factor-1, and vimentin) on small biopsies of non-small cell lung cancer effectively parallels the corresponding profiling and eventual diagnoses on surgical specimens," Journal of Thoracic Oncology, vol. 6, no. 6, pp. 1039-1049, 2011.

[35] A. Kargi, D. Gurel, and B. Tuna, "The diagnostic value of TTF1, CK 5/6, and p63 immunostaining in classification of lung carcinomas," Applied Immunohistochemistry and Molecular Morphology, vol. 15, no. 4, pp. 415-420, 2007. 


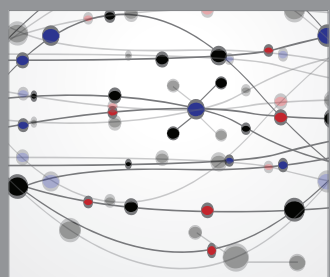

The Scientific World Journal
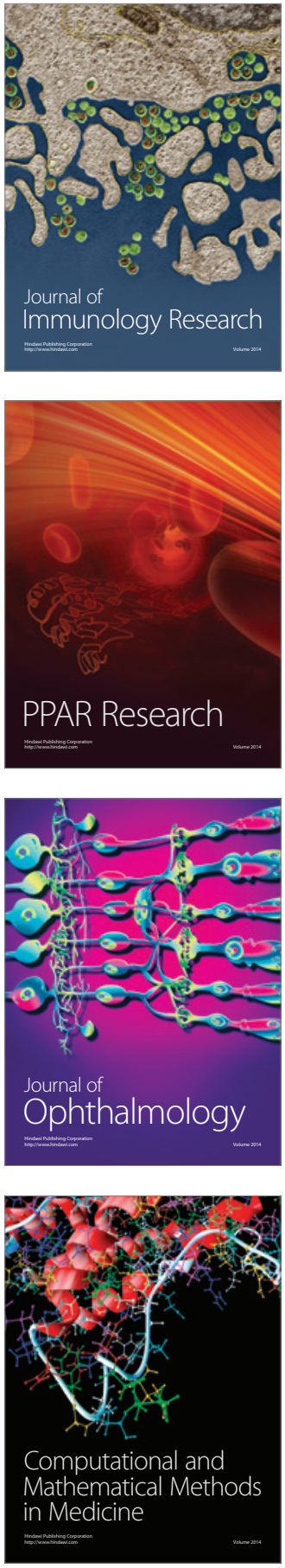

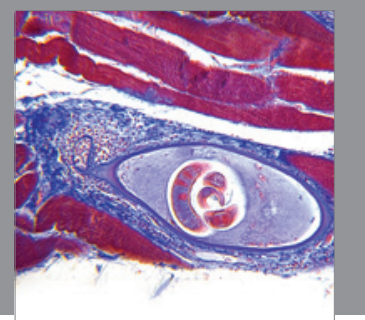

Gastroenterology

Research and Practice
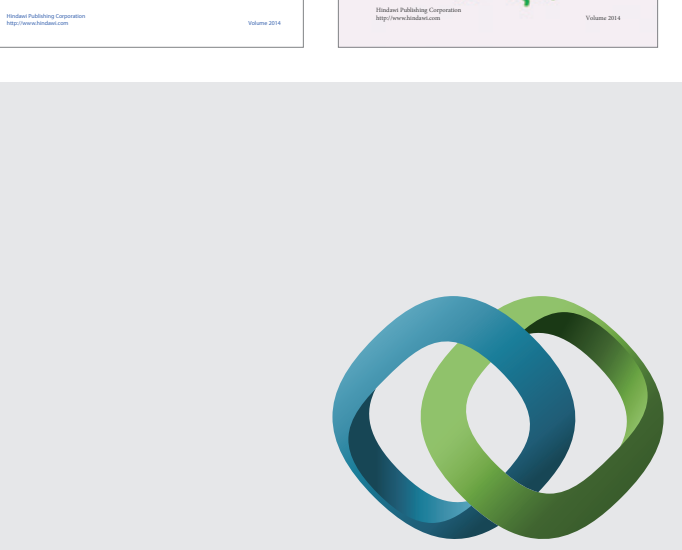

\section{Hindawi}

Submit your manuscripts at

http://www.hindawi.com
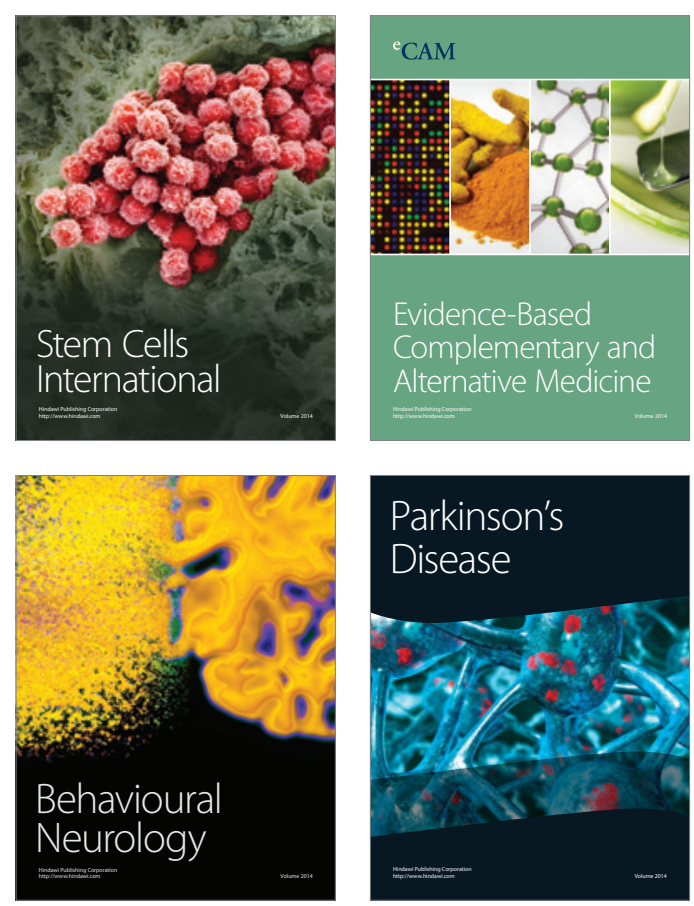

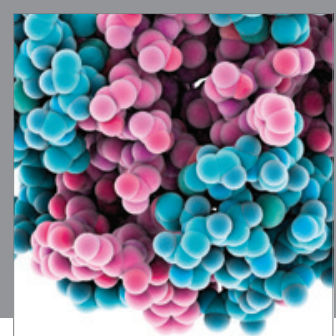

Journal of
Diabetes Research

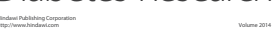

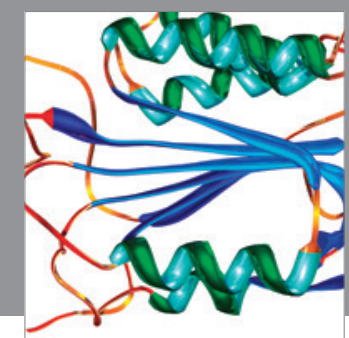

Disease Markers
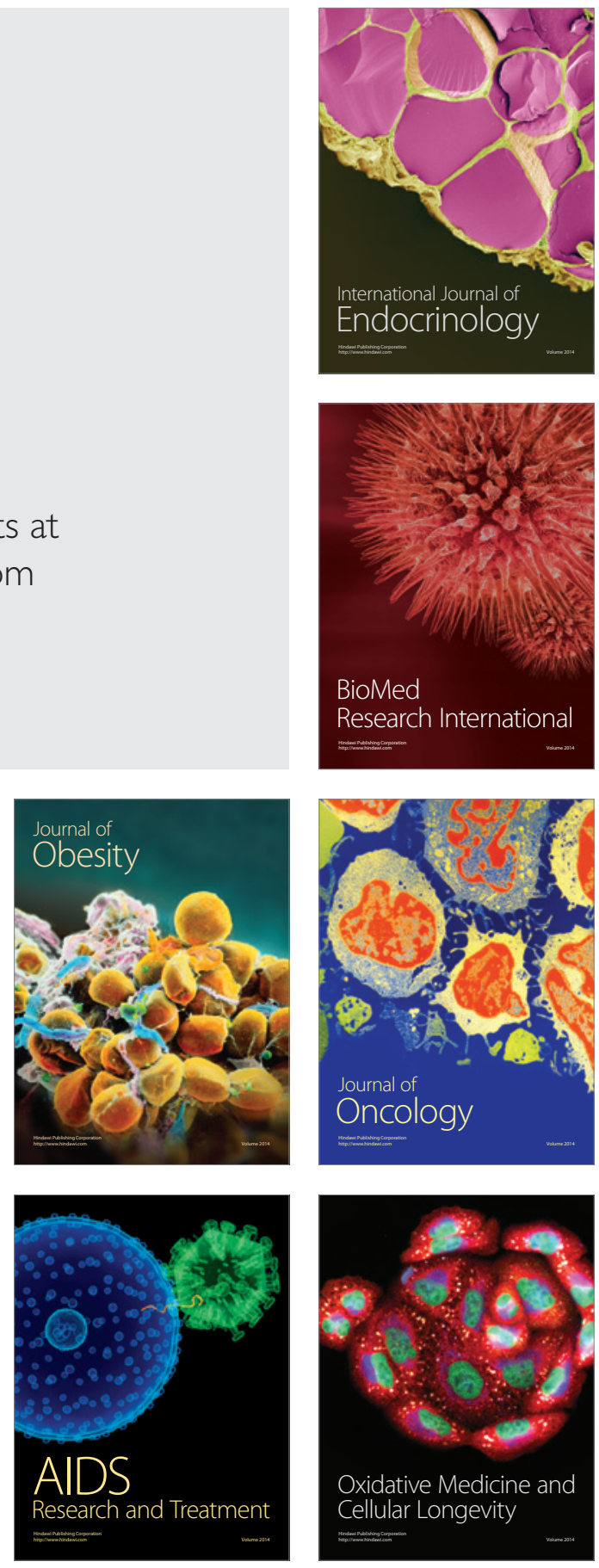